\title{
¿Conozco mi cuerpo? Estudio diagnóstico para la preparación y desarrollo cognitivo y motor en niños de infancia preescolar
}

\author{
Do I know my body? Diagnostic study for the \\ preparation and cognitive and motor \\ development in preschool children
}

\section{Sei se o meu corpo? Estudo de diagnóstico para a Elaboração $\in$ desenvolvimento cognitivo e motor de crianças pré-escolares}

\author{
Mirta Hernández Escalona? \\ Centro de Referencia Latinoamericano para la \\ Educación Preescolar (CELEP), La Habana, Cuba \\ Odet Noa Cománs ${ }^{3}$ \\ Centro de Referencia Latinoamericano para la \\ Educación Preescolar (CELEP) \\ Universidad de la Habana, La Habana, Cuba.
}

1 Artículo de investigación derivado del proyecto de investigación titulado El plan educativo para la Educación Preescolar, que lleva a cabo el Centro de Referencia Latinoamericano para la Educación Preescolar (CELEP) y el Ministerio de Educación de la República de Cuba (MINED) (2013-2015).

2 La Habana. Cuba. Licenciada en Cultura Física. Actualmente se desempeña como asesora e investigadora del Centro de Referencia Latinoamericano para la Educación Preescolar (CELEP). Ha participado en la realización de múltiples asesorías dentro y fuera del país, así como en investigaciones y publicaciones llevadas a cabo por el Ministerio de Educación de la República de Cuba. E-mail: mirta@celep.rimed.cu

3 Licenciada en Psicología. Actualmente se desempeña como asesora e investigadora del Centro de Referencia Latinoamericano para la Educación Preescolar (CELEP). Imparte docencia en la Facultad de Psicología de la Universidad de la Habana. Ha participado en investigaciones y publicaciones realizadas por el CELEP. E-mail: odet@celep.rimed.cu 
Para citar este artículo: Hernández, M. y Noa, O. (2014). ¿Conozco mi cuerpo? Estudio diagnóstico para la preparación y desarrollo cognitivo y motor en niños de infancia preescolar. Itinerario Educativo, (64), 177-192

Resumen. Los adultos deben conocer como orientar al niño e invitarlo a la realización de acciones motrices que pueda cumplir, para lograrlo es necesario diagnosticar el nivel de conocimientos que poseen los niños y las niñas sobre algunas partes de su cuerpo, así como sus relaciones en el espacio, el tiempo y el movimiento. El estudio realizado tuvo lugar en el contexto educativo cubano, por tanto tiene como referencia las características de los niños y las niñas de esta región; sin embargo pretende contribuir al enriquecimiento del pensamiento pedagógico de los educadores de cualquier lugar del mundo, pues les muestra cómo encausar adecuadamente la dirección de un proceso educativo que contribuya positivamente a la ejecución de la actividad motriz.

Palabras clave. Cuerpo, actividad motriz, preescolar (Tesauro Unesco).

Abstract. Adults should know how to orient children and encourage him to the realization of body movements that he can accomplish. To achieve this, it is necessary to diagnose the level of knowledge that children have about the different parts of their body as well as their relation to space, time and movement. The research has been carried in the Cuban educative context. Consequently, features of children in this region were taken as reference. However the research is intended to contribute to the enrichment of the pedagogical thinking of educators around the world by showing them how to lead the educative process adequately so that contribute positively to the motor development of children.

Keywords. Body, motor, preschool activity (Unesco Thesaurus).

Resumo. Os adultos devem saber como orientar a criança e convidá-lo para a realização de ações motoras você pode realizar, para alcançar isto é necessário para diagnosticar o nível de conhecimento possuído por crianças sobre as suas partes do corpo e suas relações no espaço , tempo e movimento. O estudo foi realizado no contexto educacional cubano é referenciado por ambas as características das crianças desta região; no entanto tem como objetivo contribuir para o enriquecimento do pensamento pedagógico de educadores em todo o mundo, mostrando- 
lhes como canalizar corretamente o sentido de um processo educativo que contribui positivamente para o desempenho da atividade motora.

Palavras-chave. Corpo, motor, atividade pré-escolar (Unesco Thesaurus).

\section{Introducción}

¿Cómo llegamos a la necesidad del estudio?

Los niños de la infancia preescolar han alcanzado un desarrollo motor que les permite resolver diferentes tareas y dar solución a pequeños problemas de la vida cotidiana guiados por los adultos y en relación con sus coetáneos.

Para el cumplimiento de los objetivos relacionados con el desarrollo de la flexibilidad se hace necesario, como parte de la dirección pedagógica, la utilización de imágenes y el establecimiento de relaciones entre los diferentes segmentos corporales y su movimiento por medio del "juego", de manera que se mantenga la motivación y la actividad motriz deseada para el logro de dicho objetivo.

Por otra parte, la dirección del proceso educativo debe garantizar la participación activa y consciente de los pequeños en la solución de las tareas motrices, en lo que juega un papel esencial la comunicación como medio para lograr una adecuada orientación y comprensión de la tarea.

La observación de actividades motrices nos permitieron considerar que fundamentalmente en los desplazamientos y ejercicios para el desarrollo físico general, faltaba comprensión por parte de los niños de la tarea motriz sugerida por el adulto debido al no reconocimiento o identificación de algunas de las partes del cuerpo involucradas en la tarea, la posición inicial o su variación, la relación entre el punto de partida y final del movimiento y la orientación espacial, entre los elementos más comunes que dificultaban la cooperación del niño, no fluía la actividad motriz, haciendo más lenta e ineficiente la respuesta esperada y como consecuencias de ello, el propio desarrollo de los niños y las niñas. Esta situación determinó la necesidad de explorar sobre el conocimiento que los niños poseían de algunas partes del cuerpo. 
¿Por qué se exploran estas y no otras partes del cuerpo?

A partir de la decisión de explorar, se realizó un análisis de los ejercicios y la denominación de las partes del cuerpo más usadas cotidianamente en la actividad motriz, además uno de los objetivos del programa de Conocimiento del Mundo Natural ${ }^{4}$ expresa "diferenciar las partes del cuerpo humano y las acciones que se pueden realizar con ellas". Ello nos permitió realizar la selección de aquellos segmentos corporales que en la práctica no eran del dominio de los niños para diagnosticar el estado real de su conocimiento, por medio del "juego de nombrar las partes del cuerpo"

\section{¿Qué objetivo persigue este estudio?}

Este estudio pretende diagnosticar el conocimiento que poseen los niños y niñas de 4to año de vida sobre las partes de su cuerpo y sus relaciones, en función de facilitar la dinámica de la actividad de Motricidad, el desarrollo motor en general y de la flexibilidad en particular.

¿Por qué se hace referencia al desarrollo cognitivo y motor?

El objetivo de este estudio conlleva un fin. Consiste en lograr que niños y niñas, a partir de un estado inicial diagnosticado, se apropien de las denominaciones adecuadas de algunas partes de su cuerpo y sus relaciones. De esta manera aumentarán sus conocimientos, por lo que serán seres humanos cognitivamente mejores y fluirá con éxito la actividad motriz, siendo cualitativamente superior.

El ser humano es un ser biopsicosocial y en la medida en que el niño y la niña conozcan las partes de su cuerpo y todo lo que pueden hacer relacionando unas con las otras, serán capaces de comprender, reflexionar y orientarse adecuadamente hacia la actividad, el ejercicio que se les propone, realizarlo de manera práctica, disfrutarlo, "sentir" el movimiento, su alcance, amplitud, utilidad para la vida y poder expresar y valorar el resultado alcanzado.

4 Denominación que recibe una de las áreas del desarrollo comprendidas en el programa educativo de la Educación Preescolar cubana. 


\section{Desarrollo}

¿Qué nos propusimos?

Se realizaron dos pruebas diagnósticas con un mismo contenido: una antes de realizar las actividades motrices utilizando las denominaciones adecuadas $^{5}$ para las partes del cuerpo seleccionadas y otra posterior a los dos meses en que se realizó la experiencia con las educadoras, niñas y niños. De esta manera se compararía el desarrollo cognitivo y motor de los niños y niñas.

Se aplicaron diez comprobaciones, a las siguientes partes del cuerpo:
- $\quad$ cara
- costado
- barbilla
- glúteos
- $\quad$ pecho
- muñecas
- hombros
- tobillos
- $\quad$ nuca
- talones

La concepción metodológica para la realización de la prueba fue la siguiente:

- Aplicarla individualmente, si fuera posible no mezclar los niños/ niñas que la realizaban con los que no la habían realizado.

- Conversar con el niño/a, explicarle que «vamos a jugar con los nombres de las partes del cuerpo»

- Señalar cada parte del cuerpo una a una y preguntarle el nombre de dicha parte. De obtener resultados positivos, es decir, EL NIÑO/A NOMBRA LA PARTE DEL CUERPO, registrar el resultado (N) y continuar con otra.

- En caso de no nombrar, preguntar sobre otras partes evidentemente conocidas por el niño para evitar el fracaso ante la tarea, luego insistir en el nombre o aplicar un nivel de ayuda para lograr al menos

5 Es necesario aclarar que las denominaciones adecuadas han sido estudiadas según el contexto cubano, pero en otro contexto las denominaciones pueden cambiar, lo cual no significa que sean inadecuadas. 
el RECONOCIMIENTO como: ¿̇cuál es «Xxxx»? y mencionar el nombre de la misma. Si la reconoce, señala, registrar el resultado (R)

- $\quad$ Si el niño/a no reconoce la parte señalada se le nombra y se registra el resultado (?)

\section{¿Cómo se aplicó?}

Para lograr un proceso de aplicación satisfactorio de las dos pruebas diagnósticas y para implementar el uso verbal de las partes del cuerpo en las actividades motrices durante dos meses, fue necesario preparar a las educadoras de este año de vida.

En la preparación se contó con la participación de 7 educadoras, las 7 subdirectoras de los círculos infantiles ${ }^{6}$ correspondientes y las 6 metodólogas municipales ${ }^{7}$

El criterio de selección que se sostuvo para determinar los centros y su personal docente, fue el de la voluntariedad a participar, el compromiso personal con la sistematicidad y calidad requerida en la realización de la tarea y la responsabilidad con la entrega de la información solicitada.

Durante la preparación de estas educadoras, subdirectoras y metodólogas, se realizó un diagnóstico inicial para valorar sus conocimientos sobre la capacidad y habilidades profesionales para estimularla. De acuerdo al resultado obtenido se realizó una preparación teórica, técnica, metodológica y práctica. En las primeras sesiones de preparación ellas mismas asumían el rol de los niños para imaginar la reacción de estos y a la vez se entrenaban en las actividades a realizar como educadoras, esto facilitó la dinámica y la concientización del problema para ellas.

A continuación se efectuaron actividades demostrativas con los propios niños y niñas que fueron observadas y analizadas por las participantes.

6 Denominación que reciben las instituciones infantiles cubanas.

7 Denominaciones que reciben el personal encargado de asesorar y supervisar el proceso educativo a nivel micro. 
A partir de entonces se inició la puesta en práctica de la experiencia en la Gimnasia Matutina, Educación Física y Actividad Motriz Independiente de cada grupo y se realizaron observaciones a la aplicación de las pruebas y al desarrollo de estas actividades motrices durante el período de dos meses por parte de las subdirectoras y metodólogas, derivando las acciones metodológicas correspondientes para perfeccionar el trabajo.

Durante el proceso de aplicación de la prueba inicial se notó que:

- Los niños y las niñas participan con agrado en el "juego de nombrar las partes del cuerpo"

- Generalmente los que nombraron el área correspondiente a la barbilla lo hicieron con la denominación "mango", lo cual NO fue aceptado como válido.

- De igual modo, los que nombraron el área correspondiente al costado lo realizaron con la denominación "lado" y fue aceptado como correcto.

- Asimismo, los que nombraron el área correspondiente a los glúteos lo hicieron con la denominación "nalgas", aceptado como adecuado. Hubo otras denominaciones no aceptadas como: fondillo, fotingo, cucu, culo. En este caso se aprecian algunos rasgos "prejuiciosos y/o suspicaces" en manifestaciones comportamentales de los niños y las niñas al preguntarle el nombre de esta parte del cuerpo, tales como: no querer nombrar porque es "una mala palabra", sonreír, taparse la boca, bajar la cabeza, actitudes que indican haber sido trasmitidas por los adultos más cercanos, pues al final de la experiencia se asume por la mayoría de los niños el término glúteos y se utilizó con naturalidad.

- $\quad$ Finalmente, los que nombraron el área correspondiente a los talones lo realizaron con la denominación "calcañar", la misma fue aceptada como correcta. 
Tabla 1.

Muestra los resultados de la prueba diagnóstica inicial:

\section{Niños y niñas que nombran las partes del cuerpo}

4to. Año

de Vida
TOTAL DE NI-

ÑOS Y NIÑAS: 137

\begin{tabular}{|c|c|c|c|c|c|c|c|c|c|c|}
\hline & 胥 & 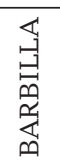 & 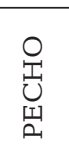 & 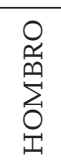 & $\begin{array}{l}\text { U } \\
\text { 己 } \\
\text { Z }\end{array}$ & 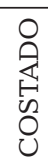 & 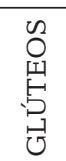 & 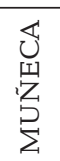 & 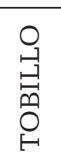 & 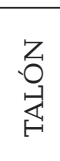 \\
\hline 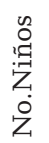 & 61 & 6 & 37 & 22 & 3 & 9 & 52 & 5 & 2 & 2 \\
\hline$\%$ & 44,5 & 4,4 & 27,0 & 16,1 & 2,2 & 6,6 & 38,0 & 3,6 & 1,5 & 1,5 \\
\hline
\end{tabular}

Las partes del cuerpo más nombradas en la prueba inicial fueron: la cara, 61 niños $(44,5 \%)$, las nalgas o glúteos fueron nombradas por 52 niños para el 38\% y el pecho, 37 niños con el $27 \%$.

Las partes del cuerpo menos nombradas en la prueba inicial fueron: el tobillo y el talón, solo por 2 niños para el 1,5\% y tres pequeños nombraron la nuca, representando el 2,2\% del total.

A partir la aplicación del diagnóstico inicial, se inició el trabajo con subdirectoras, educadoras, niños y niñas. Se realizaron ejercicios variados y novedosos durante la ejecución de los desplazamientos, ejercicios para el desarrollo físico general, ejercicios principales, juegos de movimiento y recuperación. Se cumplieron indicaciones metodológicas específicas relacionadas con el orden de los ejercicios, su combinación, ritmo, momentos más propicios, métodos para el desarrollo de la flexibilidad y la postura, entre otras.

Las propuestas, además del desarrollo de la flexibilidad, propiciaron en los niños y niñas el conocimiento de las diferentes partes del cuerpo y 
sus posibilidades individuales, la relación cuerpo-movimiento-espaciotiempo, favoreciendo el desarrollo de la coordinación, orientación en el espacio, la anticipación y regulación del movimiento como capacidades coordinativas, así como la postura correcta. Se realizaron combinaciones de 2 ejercicios que relacionan las mismas partes del cuerpo, pero con diferente dirección e influencia de grupos musculares y articulaciones.

Figura 1

Muestra ejemplos de ejercicios

Ejemplos:

- Acercar la oreja al hombro y luego los hombros a las orejas.

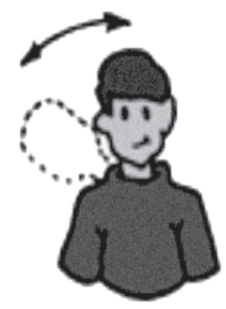

- Barbilla a al pecho y barbilla al hombro.

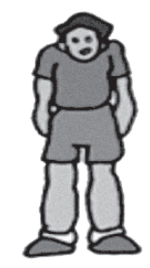

Figura 2

Muestra ejemplos de ejercicios
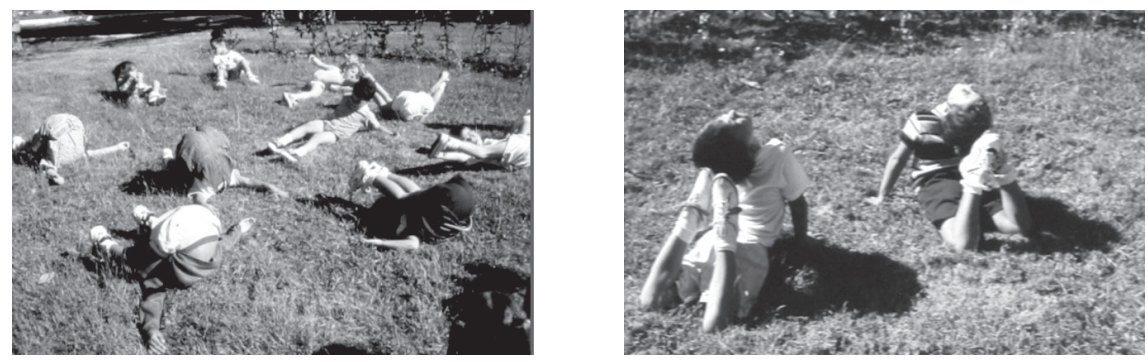

ITINERARIO EDUCATIVO • ISSN OIZI-Z753 • AÑO XXVIII, N. ${ }^{\circ} 64$ • JULIO - DICIEMBRE DE 2OI4 • P. I77-I92 
Los pies tocan la cabeza...luego se les propone hacer lo mismo pero boca abajo: "iquién puede tocar ahora, la cabeza con los pies?" Estos ejercicios se combinan, invitando a la misma relación de los pies y la cabeza pero desde diferente posición, de este modo trabajan grupos musculares antagónicos que se compensan, equilibran su esfuerzo y por lo tanto garantizan la adecuada postura.

Dichas combinaciones, además de producir diferentes influencias musculares y motrices, hacen que el niño y la niña identifiquen y se concentren en cuáles son las partes relacionadas, desde que posición y en qué dirección se producirá el movimiento y posteriormente realizar la relación a la que se le invita, con la obvia influencia en el proceso de adquisición de nuevos conocimientos, el desarrollo de habilidades intelectuales, y de los diferentes procesos psíquicos.

Figura 3

Muestra movimiento de la cabeza
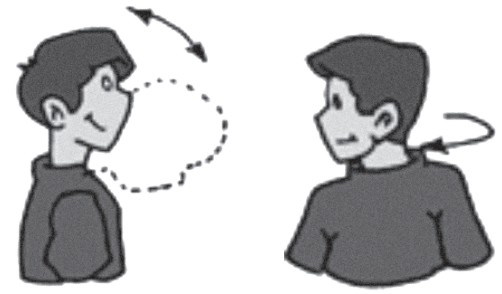

También se les proponía que observaran cómo y hasta dónde se desplazaba cada segmento de su cuerpo, lo que les permitió conocerse y regular mejor los movimientos, apreciar sus posibilidades reales para desde ahí, trazarse mayor alcance en la amplitud. Ello fue posible porque ya en esta edad comienzan a manifestarse intereses alrededor del resultado, la valoración positiva que desea tener del adulto y la significación que tiene para ellos realizarlo "bien".

Se les sugería que "SINTIERAN" el movimiento y lo expresaran verbalmente: qué parte o región del cuerpo se estira en un movimiento, qué sensación les produce este estiramiento, qué relación y dirección y cuáles en otra, cuáles se unen, cómo se agrupan, hacia dónde va el peso del cuerpo, que sienten cuando caminan con un pequeño objeto sobre la cabeza, entre otras. 
Estos elementos nos permitieron valorar en qué medida se producía la influencia de los ejercicios sobre las diferentes partes del cuerpo pues la posibilidad de verbalizar brevemente qué se siente, es un indicador importante a tener en cuenta para avanzar o no en nuevas pequeñas metas en cuanto a amplitud del movimiento protegiendo la integridad física de los niños y niñas. De igual modo contribuye a la valoración, por parte del educador, del nivel de apropiación del nombre de los diferentes segmentos corporales. Como puede apreciarse en este proceso se integra lo motriz, lo cognitivo y lo afectivo.

El juego corporal y la propuesta de pequeñas situaciones problémicas también formó parte de las formas de dirección del proceso educativo, pues mantienen la motivación, enriquecen el conocimiento de la partes del cuerpo, sus funciones y relaciones; facilita la atención y en consecuencia la percepción y representación del movimiento; desarrolla el pensamiento pues el pequeño debe analizar primero qué se le propone y luego realizar la acción a partir de la relación a la que se le invita.

Por ejemplo:

¿Cómo llevar los talones a las nalgas?

¿Cómo llevar las nalgas o glúteos a los talones?

¿Cómo llevar el pecho al piso?..."El sapito"

Figura 4

Muestra la ejecución de los juegos corporales
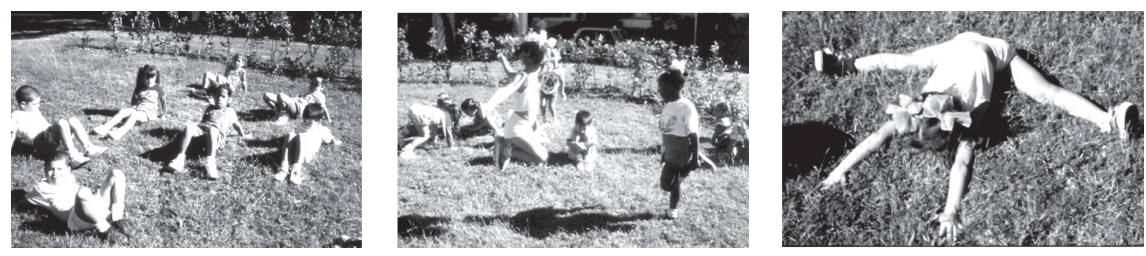

Estas situaciones de juego corporal y de solución de pequeños problemas motrices, además de contribuir al desarrollo de la flexibilidad, aumentan las vivencias de los niños, aspectos esenciales para su desarrollo.

ITINERARIO EDUCATIVO • ISSN OIZI-Z753 • AÑO XXVIII, N. 64 • JULIO - DICIEMBRE DE 20I4 • P. I77-I92 
También las educadoras preguntaban cómo acercar, separar, relacionar diferentes partes del cuerpo en una posición, en otra, para hacerlo cada vez de un modo diferente o sugerían, proponían opciones en caso necesario para enriquecer las vivencias motrices de los pequeños y lograr el cumplimiento de sus objetivos.

Las rimas y canciones formaron parte de las disímiles formas de dirigir el proceso educativo.

Ejemplo: De pie con los brazos abajo, realizar flexión lateral del tronco rozando con la mano el muslo y la pierna, regresar a la posición inicial posterior a cada flexión. Con una sencilla rima sería:

"Toco el muslo

(y regresar a la posición inicial)

ahora la rodilla

(y regresar)

una vez más... iY me pegué! (esperar algunos segundos),

llego más abajo y despacito regresé" (volver a la posición inicial)

De este modo se lograba combinar ejercicios activos dinámicos con los estáticos, esenciales para el desarrollo de la flexibilidad, a la vez que reafirma el conocimiento de las diferentes partes del cuerpo de un modo eminentemente práctico.

Otros ejemplos de relaciones que se establecieron:

"¿cómo tocar el piso con las manos sin agacharnos?"

"¿̇cómo el pecho toca los muslos?"

"¿̇cómo llevar la nariz a las rodillas?"

"¿cómo tocamos las rodillas con la frente?",

"iAgarramos los talones y nos peinamos con los pies!"

Figura 5

Muestra ejecución ejemplos
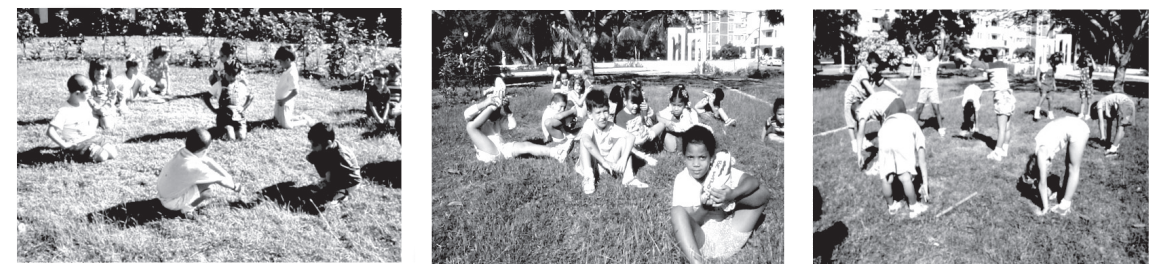
Una vez concluidos los dos meses de trabajo se realizó la segunda prueba diagnóstica a los 137 niños y niñas y se compararon sus resultados con el diagnóstico inicial.

Tabla 2

Muestra los resultados sobre el conocimiento de las partes del cuerpo de mayor interés para la experiencia en la segunda prueba diagnóstica

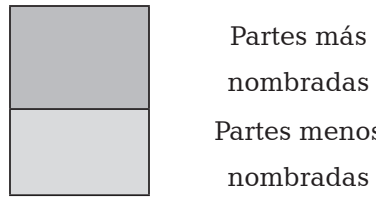

\begin{tabular}{|c|c|c|c|c|c|c|c|c|c|c|}
\hline & 奈 & 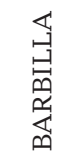 & 茎 & 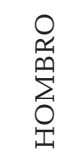 & $\begin{array}{l}\text { U } \\
\text { 己 } \\
\text { 乙 }\end{array}$ & 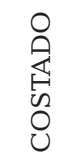 & 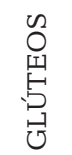 & 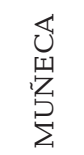 & 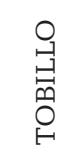 & $\begin{array}{l}Z \\
\text { 芯 } \\
\text { 芯 }\end{array}$ \\
\hline 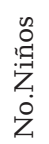 & 124 & 110 & 116 & 97 & 77 & 59 & 106 & 62 & 58 & 75 \\
\hline$\%$ & 90,5 & 80,3 & 84,7 & 70,8 & 56,2 & 43,1 & 77,4 & 45,3 & 42,3 & 54,7 \\
\hline
\end{tabular}

Las partes del cuerpo más nombradas fueron: La cara, pecho y barbilla con 124 niños (90,5\%), $116(84,7 \%)$ y 110 (80,3\%) respectivamente.

Las partes del cuerpo menos nombradas fueron: el tobillo, 50 niños (42,3\%), el costado con 59 niños para el 43,1\% y la muñeca, 62 niños $(45,3 \%)$

\section{Conclusiones}

Se obtuvo como resultado final de la experiencia que de 137 niños y niñas, 123 de ellos, el 89,8\% lograron nombrar o reconocer TODAS las partes del cuerpo exploradas. Sólo 14 niños no lograron este resultado en una o algunas partes.

Se observó que las educadoras se apropiaron de los términos para su uso cotidiano, pues representaba para ellas mayor cientificidad, creci- 
miento profesional y personal. En este sentido el estudio funcionó como superación para ellas.

El estudio logró mayor desarrollo cognitivo tanto en niños como en las educadoras.

El estudio contribuyó al desarrollo de habilidades como: comprensión, solución de situaciones problémicas motrices, orientación espacial, pues a través del diseño de las tareas aplicadas los niños y niñas tenían que pensar en las relaciones posibles entre las partes del cuerpo, no solo reproducir o simular para desempeñar la actividad motriz.

Facilitó el desarrollo motor pues se notó que la actividad motriz fluía mucho más cuando los niños eran capaces de seguir las orientaciones verbales conociendo las denominaciones adecuadas y las relaciones planteadas.

El proceder en la prueba contribuyó a la disminución del fracaso ante la tarea, en cada pregunta la respuesta de los niños y niñas era permitida y solo con niveles de ayuda se logró el máximo desarrollo posible.

Se demostró que para lograr un desarrollo motor real no basta con la orientación y respuesta verbal de la tarea, es necesario "hacer con las partes del cuerpo", lo cual significa realizar el movimiento conociendo las partes del cuerpo y sus relaciones.

\section{Bibliografía}

Alonso (2006). El impacto sobre el hombre de la evolución de los sistemas hombre-técnica-ambiente. Disponible en www.monografías.com/ trabajos15/ergonomía/ergonomía.shtml

Alter (2001). Los estiramientos. Bases Científicas y desarrollo de ejercicios. Barcelona, España: Editorial Paidotribo.

Colectivo de autores. (1995) En torno al Programa de Educación Preescolar. La Habana, Cuba: Editorial Pueblo y Educación.

Colectivo de autores. (1998). Programa educativo para la educación preescolar. Primero al 6to. Año de Vida. La Habana, Cuba: Editorial Pueblo y Educación. 
De Vega. (2005). Introducción a la Psicología Cognitiva. Tomo II. La Habana, Cuba: Editorial Félix Varela.

Di Santo (2006). Entrenamiento de la Flexibilidad. Instituto del Profesorado en Educación Física. Disponible en www.sobreentrenamiento.com

Di Santo (2006). Importancia de la flexibilidad. Disponible en http://www. squashglobal.com/Artículos3.html

Di Santo (2006). La flexibilidad en las distintas edades de la vida. Disponible en http://www.sobrentrenamiento.com

Engels (2002). Dialéctica de la naturaleza. La Habana, Cuba: Editorial Pueblo y Educación.

Esper Di Cesare (2006). El entrenamiento de la flexibilidad muscular en las divisiones formativas de baloncesto. En Revista Digital. Buenos Aires. Disponible en www.efdeportes.com/efd0/b-basq.htm-10k

Fariñas. (2002). Maestro para una didáctica del aprender a aprender. La Habana, Cuba. Editorial Félix Varela.

Fernández (2006). La flexibilidad en las artes marciales. Disponible en http://www.shotokanryukaseha.com/articulos/la_flexibilidad.htm\#9

García (2009). Alternativas Metodológicas para favorecer la capacidad física de flexibilidad en los niños y niñas del grado preescolar. Las Tunas. Cuba. Tesis en opción al Título de Máster en Ciencias de la Educación.

Hedrick (2006). Entrenamiento de Flexibilidad para Incrementar el Rango de Movimiento. Disponible en www.sobreentrenamiento.com

Ibañez y Torrebadella (1989) 1.004 Ejercicios de Flexibilidad. Barcelona, España: Editorial Paidotribo.

Le Boulch (2002). El movimiento en el desarrollo de la persona. Barcelona, España: Editorial Paidotribo.

Legaspi de Arismend. (1999). Pedagogía Preescolar. La Habana, Cuba: Editorial Pueblo y Educación.

López (2001). Aproximación a un nuevo concepto de Educación Integral. La Habana, Cuba: Editorial Félix Varela. 
López y Arias (2002). Teoría vigotskiana del desarrollo infantil. La Habana, Cuba. Curso Pre-Evento. V Encuentro Internacional de Educación Inicial y Preescolar.

López y Siverio (2005). El proceso educativo para el Desarrollo Integral de la Primera Infancia. . La Habana, Cuba: Editorial Félix Varela.

Meinel (1977). Didáctica del Movimiento. La Habana, Cuba: Editorial Orbe.

Norris (2000). La flexibilidad. Principios y práctica. Barcelona, España: Editorial Paidotribo.

Platónov (1983). Metodología del desarrollo de la flexibilidad. Aspectos y estructura de la flexibilidad. Rusia: Editorial Zdorovia.

Ruiz (1989). Gimnasia Básica. La Habana, Cuba: Editorial Pueblo y Educación.

Ruiz, López y Dorta (1985). Metodología de la enseñanza de la Educación Física. La Habana, Cuba: Editorial Pueblo y Educación.

Ruiz (1987). Desarrollo motor y actividades físicas. Madrid, España: Editorial Gymnos.

Saavedra (1997). Ejercicio físico y medicina preventiva. Mecanismos de adaptación fisiológica. Conferencia Latinoamericana de promoción de la salud. Santiago, Chile. Disponible en www.efdeportes.com/ efd7/saav72.htm 